\title{
Weak Decays of Heavy Mesons in the Instantaneous Bethe Salpeter Approach
}

\author{
G. Zöller, S. Hainzl, C.R. Münz \\ Institut für Theoretische Kernphysik der Universität Bonn, \\ Nußallee 14-16, 53115 Bonn, FRG \\ M. Beyer \\ Institut für Theoretische Kernphysik der Universität Bonn, \\ Nußallee 14-16, 53115 Bonn, FRG \\ and \\ Max Planck Gesellschaft, AG 'Theoretische Vielteilchenphysik' \\ Universität Rostock, Universitätsplatz 1, 18055 Rostock, FRG
}

\begin{abstract}
In the framework of the instantaneous Bethe Salpeter equation we investigate weak decays of $B$ and $D$ mesons. Mesons are described as $q \bar{q}$ states interacting via a mixture of a scalar and a vector confining kernel and a one gluon exchange. The model parameters are fixed by a fit to the meson mass spectrum including also the light mesons. We calculate form factors and compare our results to the pole dominance hypothesis. From a fit to ARGUS and CLEO data on $B \rightarrow D^{*} \ell \nu$ semileptonic decay we extract the Cabbibo Kobayashi Maskawa matrix element to be $V_{c b}=(0.032 \pm 0.003)\left(1.49 p s / \tau_{B}\right)^{1 / 2}$. The Isgur Wise function is calculated utilizing the heavy quark mass limit. Finally, we give some results on non-leptonic decays.
\end{abstract}

FAX 0381-4982857, Email:beyer@darss.mpg.uni-rostock.de BONN TK-94-15, MPG-VT-UR-44/94 


\section{Introduction}

In recent years the decay of $B$ and $D$ mesons has become one of the most exciting source of information on fundamental interactions and symmetries. More experiments are expected, since the plans for future $B$-meson factories are now beginning to be realized.

Theoretical effort to extract the fundamental quantities relevant on the 'quark level' from the 'hadronic counting rates' has been enormous and also successful. The ultimate aim is to extract those quantities model independent. On the other hand, interpretation in terms of model degrees of freedom has been proven very useful: Since QCD and confinement is still not solved at moderate energies, weak decays may be used to improve on the dynamical description of the underlying quark structure and therefore to extract information on the relevant degrees of freedom.

Considerable amount of effort has been put into the description of mesons in terms of the underlying quark structure. For heavy mesons, e.g. charmonium and bottomonium, non-relativistic models have been particularly successful [1]- [12]. For a review see e.g. [13, 14]. However, a closer look reveals that relativistic effects are non-negligible even for mesons involving only heavy quarks. We found that those are particularly important for $M 1$ transitions in charmonium and even in the form factors of $B$-meson decays [15, 16]. We also found that non-covariance of the non-relativistic model may lead to inconsistencies for the $B$ decay form factors of about $5-10 \%$ [16. The non-relativistic model is worse in cases where heavyto-light or light-to-light transitions are involved. Although the mass spectrum may be reproduced, the form factors and therefore the description of the decay rates fails badly [17.

We have now chosen a quite different approach utilizing the instantaneous Bethe Salpeter equation (IBS) to treat the $q \bar{q}$ system within a relativistically covariant formalism [18]. The model is able to describe the meson mass spectrum for low radial excitations. It has been applied to the calculation of leptonic decays, viz. decay constants, $\gamma \gamma$ decays [19], and to elastic form factors of mesons [20] as well as to charmonium and bottomonium [21]. Similar lines have been followed by [22]- 26]. In the following section we will give a short survey of the model.

To calculate semileptonic decays, we first determine the two form factors of the $0^{-} \rightarrow 0^{-}$transitions and the four form factors of the $0^{-} \rightarrow 1^{-}$transitions. We compare our results to the pole dominance ansatz, which is a different way to describe the $q^{2}$ behavior of the form factors. This will be presented in section 3 .

Recently, much attention has been paid to heavy quark effective theory (HQET) (see e.g. 27] and references therein), which relates form factors of $B \rightarrow D$ to those of $B \rightarrow D^{*}$ transitions introducing heavy quark symmetries. All form factors are 
then related to one universal function (i.e. the Isgur Wise function [28]). Some of the more recent calculations utilizing the Bethe Salpeter approach are specifically dedicated to calculate this universal function [29, 30]. In section 4 we connect our results to the notion of HQET and give the result for the Isgur Wise function.

In section 5 we discuss light meson transitions. We compare the BetheSalpeter approach to the non-relativistic ansatz. However, since we use an instantaneous kernel and ladder approximation, results for the light mesons should be less reliable as for the heavy mesons.

Section 6 is dedicated to non-leptonic decays of heavy mesons. The discussion on QCD corrections in the operators, initiated by the latest CLEO results on $B$ decays is not entered here. Through being an important issue it is not considered the main subject of the present paper. For definiteness we neglect additional gluonic corrections in the weak operators.

We conclude with a summary of our main results in section 7 .

\section{The quark model}

Mesons are treated as $q \bar{q}$ states in the framework of the instantaneous Bethe Salpeter (BS) equation. A more detailed description of the model used here, has been given in [18, 19] in a different context, and references therein. Here we summarize the main results relevant for weak decays of mesons.

For an instantaneous Bethe Salpeter kernel and utilizing free quark propagators with effective quark masses $m_{1}$ and $m_{2}$ one may perform the $p^{0}$ integrals of the BS-equation. This is done in the rest frame of the bound state with mass $M$ and lead to the (full) Salpeter equation, viz.

$$
\begin{aligned}
\Phi(\boldsymbol{p}) & =\int \frac{d^{3} p^{\prime}}{(2 \pi)^{3}} \frac{\Lambda_{1}^{-}(\boldsymbol{p}) \gamma^{0}\left[V\left(\boldsymbol{p}, \boldsymbol{p}^{\prime}\right) \Phi\left(\boldsymbol{p}^{\prime}\right)\right] \gamma^{0} \Lambda_{2}^{+}(-\boldsymbol{p})}{M+\omega_{1}+\omega_{2}} \\
& -\int \frac{d^{3} p^{\prime}}{(2 \pi)^{3}} \frac{\Lambda_{1}^{+}(\boldsymbol{p}) \gamma^{0}\left[V\left(\boldsymbol{p}, \boldsymbol{p}^{\prime}\right) \Phi\left(\boldsymbol{p}^{\prime}\right)\right] \gamma^{0} \Lambda_{2}^{-}(-\boldsymbol{p})}{M-\omega_{1}-\omega_{2}}
\end{aligned}
$$

with

$$
\Phi(\boldsymbol{p})=\left.\int \frac{d p^{0}}{(2 \pi)} \chi_{P}\left(p^{0}, \boldsymbol{p}\right)\right|_{P=(M, \mathbf{0})}
$$

where $\chi_{P}\left(p^{0}, \boldsymbol{p}\right)$ is the full Bethe-Salpeter amplitude. Here $\omega_{i}=\sqrt{\boldsymbol{p}^{2}+m_{i}^{2}}$, and we introduce energy projection operators $\Lambda_{i}^{ \pm}(\boldsymbol{p})=\left(\omega_{i} \pm H_{i}(\boldsymbol{p})\right) /\left(2 \omega_{i}\right)$ in obvious notation, where $H_{i}(\boldsymbol{p})=\gamma^{0}\left(\boldsymbol{\gamma} \cdot \boldsymbol{p}+m_{i}\right)$ is the standard Dirac Hamiltonian (see e.g. refs.[18, 19]).

The dynamical input of the model is defined by a confinement plus one gluon exchange (OGE) kernel, $V=V_{C}+V_{G}$. Confinement is introduced as a mixture 
of a scalar and a vector type kernel, viz.

$$
\left[V_{C}\left(\boldsymbol{p}, \boldsymbol{p}^{\prime}\right) \Phi\left(\boldsymbol{p}^{\prime}\right)\right]=\mathcal{V}_{C}^{S}\left(\left(\boldsymbol{p}-\boldsymbol{p}^{\prime}\right)^{2}\right)\left[\Phi\left(\boldsymbol{p}^{\prime}\right)-\gamma^{0} \Phi\left(\boldsymbol{p}^{\prime}\right) \gamma^{0}\right]
$$

with a scalar function $\mathcal{V}_{C}$. The mixture of a scalar and a vector spin structure has been introduced in order to give an improved description of the spin orbit splitting. Note, that a purely scalar confining kernel leads to an RPA instability of the instantaneous Bethe Salpeter equation.

Since is it more suggestive to introduce a confining potential in co-ordinate space, we assume that the Fourier transform is given by

$$
\mathcal{V}_{C}^{F}(r)=a_{c}+b_{c} r
$$

To derive the OGE kernel, we chose Coloumb gauge for the gluon propagator. This way it is possible to retain a covariant formulation within an instantaneous treatment of the BS equation, and it allows to substitute $q^{2}$ by $-\boldsymbol{q}^{2}$. The OGE kernel then reads [24, 31]

$$
\begin{aligned}
& {\left[V_{G}\left(\boldsymbol{p}, \boldsymbol{p}^{\prime}\right) \Phi\left(\boldsymbol{p}^{\prime}\right)\right]=\mathcal{V}_{G}\left(\left(\boldsymbol{p}-\boldsymbol{p}^{\prime}\right)^{2}\right)} \\
& \quad \cdot\left[\gamma^{0} \Phi\left(\boldsymbol{p}^{\prime}\right) \gamma^{0}-\frac{1}{2}\left(\boldsymbol{\gamma} \Phi\left(\boldsymbol{p}^{\prime}\right) \boldsymbol{\gamma}+(\boldsymbol{\gamma} \hat{x}) \Phi\left(\boldsymbol{p}^{\prime}\right)(\boldsymbol{\gamma} \hat{x})\right)\right]
\end{aligned}
$$

with the operator $\hat{x}=\mathbf{x} /|\mathbf{x}|$, and

$$
\mathcal{V}_{G}\left(\boldsymbol{q}^{2}\right)=4 \pi \frac{4}{3} \frac{\alpha_{s}\left(\boldsymbol{q}^{2}\right)}{\boldsymbol{q}^{2}}
$$

For $\alpha_{s}\left(q^{2}\right)$ we make the following assumption. For $Q^{2} \equiv-q^{2}=\boldsymbol{q}^{2} \gg \Lambda_{Q C D}^{2}$ the strong coupling $\alpha_{s}\left(\boldsymbol{q}^{2}\right)$ behaves like the running coupling constant $\alpha_{s}^{\text {run }}\left(Q^{2}\right)$ of QCD, viz. 32

$$
\alpha_{s}^{\text {run }}\left(Q^{2}\right)=\frac{A}{\ln \left(Q^{2} / \Lambda_{Q C D}^{2}\right)}\left(1-B \frac{\ln \left(\ln \left(Q^{2} / \Lambda_{Q C D}^{2}\right)\right)}{\ln \left(Q^{2} / \Lambda_{Q C D}^{2}\right)}\right)+\ldots
$$

with $A=4 \pi / 9$ and $B=64 / 81$. For $Q^{2} \ll \Lambda_{Q C D}^{2}$ we assume some saturation value $\alpha_{\text {sat }}$ which will be a fit parameter of the model. In between we simply assume some smooth interpolation between the two regions.

For consistency with the confining potential, we use co-ordinate representation of the the Coulomb kernel. It may be parameterized as follows [18.

$$
\alpha_{s}(r)=\frac{A}{2 \ln \left(e^{-(\gamma+\mu a)} / a+e^{A /\left(2 \alpha_{s a t}\right)}\right)}\left[1-B \frac{\ln \left(2 \ln \left(e^{-\tilde{\mu} a} / a+e^{1 / 2}\right)\right)}{2 \ln \left(e^{-\mu a} / a+e^{B / 2}\right)}\right]
$$

where $\gamma=0.577215 \ldots$ is the Euler Mascheroni constant, and $a=r \Lambda_{Q C D}$. We set $\mu=4$ and $\tilde{\mu}=20$. 
As discussed in 18, 19 the potential needs to be regularized, which introduces an additional parameter $r_{0}$. The reason for that is different from the non-relativistic case, where the terms of order $\boldsymbol{p}^{2} / \mathrm{m}^{2}$ like the spin-spin and spinorbit interaction lead to a collapse of the wave-function at the origin. For most of the Salpeter amplitudes and a fixed coupling constant Murota [31] has explicitly shown that the amplitudes are divergent for $r \rightarrow 0$. For a running coupling constant this divergence is less pronounced, but still present. We therefore will use the following regularized potential (in co-ordinate space)

$$
\begin{aligned}
& \mathcal{V}_{G}^{F}(r)=-\frac{4}{3} \frac{\alpha_{s}(r)}{r} \quad \text { for } r>r_{0} \\
& \mathcal{V}_{G}^{F}(r)=a_{G} r^{2}+b_{G} \text { for } r \leq r_{0}
\end{aligned}
$$

where $a_{G}$ and $b_{G}$ are simply determined by the condition that $\mathcal{V}_{G}^{F}(r)$ and its first derivative are continuous functions. The dependence of $\alpha_{s}(r)$ on $\Lambda_{Q C D}$ given by eq.(8) is rather weak and may be compensated for by modifying $\mu$ and $\alpha_{\text {sat }}$. We use $\Lambda_{Q C D}=200 \mathrm{MeV}$ for our calculation. The dependence of the mass spectra on the regularization parameter $r_{0}$ is very weak so that the differences in the mass spectra calculated with the regularized and unregularized potential are quite small. For our further calculation we will take $r_{0}=0.1 \mathrm{fm}$.

To solve the Salpeter equation numerically, eq. (1) is rewritten as an eigenvalue problem, see e.g. [18, 22]. This way it is it possible to utilize the variational principle to find the respective bound states. To this end the Salpeter amplitude $\Phi$ is expanded into a reasonable large number of basis states used as a test function. As a suitable choice of basis states we have taken Laguerre polynomials and found that about ten basis states lead to sufficient accuracy, see also [18, 19].

The parameters of the model are given in Table 1. These are the quark masses, the offset $a_{c}$ and slope $b_{c}$ of the confinement interaction eq. (4) and the saturation value $\alpha_{s a t}$ for $\alpha_{s}(r)$ in eq.(8). They are determined to give a good overall description of the meson mass spectrum. The mesons relevant for semileptonic decays, and their low radiative excitations are shown in Figure 1 .

\section{Form factors and semileptonic decays}

Semileptonic decays are treated in current-current approximation. For a transition $b \rightarrow c$ the Lagrangian is given by

$$
\mathcal{L}_{c b}=\frac{G_{F}}{\sqrt{2}} V_{c b} \quad h_{c b}^{\mu} j_{\mu}
$$

with the Cabbibo-Kobayashi-Maskawa matrix element $V_{c b}$. The leptonic $j_{\mu}$ and hadronic currents $h_{c b}^{\mu}$ are defined by

$$
j_{\mu}=\bar{\ell} \gamma_{\mu}\left(1-\gamma_{5}\right) \nu_{\ell}
$$




$$
h_{c b}^{\mu}=\bar{c} \gamma^{\mu}\left(1-\gamma_{5}\right) b
$$

The relevant transition amplitudes $\left\langle D^{(*)}\left|h_{c b}^{\mu}(0)\right| B\right\rangle$ for $B \rightarrow D$ and $B \rightarrow D^{*}$ of the hadronic current can be decomposed due to the Lorentz covariance of the current, thus introducing form factors. We use a standard representation in terms of $F_{0}\left(q^{2}\right), F_{1}\left(q^{2}\right)$ for $0^{-} \rightarrow 0^{-}$transitions, and $V\left(q^{2}\right), A_{0}\left(q^{2}\right), A_{1}\left(q^{2}\right), A_{2}\left(q^{2}\right)$ for $0^{-} \rightarrow 1^{-}$transitions. The exact definitions, and further references have been given e.g. in [16]. Note that $m_{\ell}^{2} \leq q^{2} \leq q_{\max }^{2}=\left(m_{B}-m_{D^{(*)}}\right)^{2}$ due to kinematical reasons. Helicity amplitudes $H_{ \pm}$and $H_{0}$ in terms of the form factors have been given by Körner and Schuler (KS) in a series of papers [36] and are compiled by the particle data group [32]. The respective decay rates into specific helicity states $\Gamma_{ \pm}, \Gamma_{0}$ are also given in the literature, see e.g. [32].

To determine the form factors from the model, we follow the general prescription by Mandelstam [33], see e.g. [34] for a textbook treatment. The lowest order weak kernel reads (consider e.g. the anti-quark current, flavour indices suppressed)

$$
K_{w e a k}^{\mu}\left(P, q, p, p^{\prime}\right)=\gamma^{\mu}\left(1-\gamma_{5}\right)\left[S_{q}^{F}(P / 2+p)\right]^{-1} \delta\left(p^{\prime}-p-q / 2\right)
$$

where $p$ and $p^{\prime}$ denote the relative momenta of the incoming and outgoing $q \bar{q}$ pair, $q=P-P^{\prime}$ is the momentum transfer. A pictorial demonstration of this approximation is given in Figure 2. The quark Feynman propagator is denoted by $S_{q}^{F}$. The Dirac coupling to point-like particles is consistent with the use of free quark propagators. Thus the semileptonic current matrix element, e.g for $B \rightarrow D$ that needs to be calculated, reads

$$
\begin{gathered}
\left\langle D, P_{D}\left|h_{c b}^{\mu}(0)\right| B, P_{B}\right\rangle= \\
=-\int \frac{d^{4} p}{(2 \pi)^{4}} \operatorname{tr}\left\{\bar{\Gamma}_{P_{D}}(p-q / 2) S_{\bar{q}^{\prime}}^{F}\left(P_{B} / 2+p-q\right) \gamma^{\mu}\left(1-\gamma_{5}\right)\right. \\
\left.S_{\bar{q}}^{F}\left(P_{B} / 2+p\right) \Gamma_{P_{B}}(p) S_{q}^{F}\left(-P_{B} / 2+p\right)\right\}
\end{gathered}
$$

where $\Gamma_{P}(p)$ is the amputated BS amplitude or vertex function

$$
\Gamma_{P}(p):=\left[S_{q}^{F}\left(p_{q}\right)\right]^{-1} \chi_{P}(p)\left[S_{\bar{q}}^{F}\left(-p_{\bar{q}}\right)\right]^{-1}
$$

It may be computed in the rest frame from the equal time amplitude $\Phi(\boldsymbol{p})$ using the Bethe-Salpeter equation

$$
\left.\Gamma_{P}(p)\right|_{P=(M, \mathbf{0})}=\Gamma(\boldsymbol{p})=-i \int \frac{d^{3} p^{\prime}}{(2 \pi)^{4}}\left[V\left(\boldsymbol{p}, \boldsymbol{p}^{\prime}\right) \Phi\left(\boldsymbol{p}^{\prime}\right)\right]
$$

Finally, using Lorentz transformation properties, we can calculate the full BS amplitude in any reference frame as

$$
\chi_{P}(p)=S_{\Lambda_{P}} \chi_{(M, \mathbf{0})}\left(\Lambda_{P}^{-1} p\right) S_{\Lambda_{P}}^{-1}
$$


where $\Lambda_{P}$ is the pure Lorentz boost, and $S_{\Lambda_{P}}$ the corresponding transformation matrix for Dirac spinors.

Due to the reconstruction of the full Bethe-Salpeter amplitude sketched above, the transition matrix element eq. (14) is manifestly covariant.

The spin part of the current is evaluated by a standard trace technique appropriate for the particle anti-particle formalism. The radial part have been expanded in a basis of eleven Laguerre functions. The results are found to be stable within a large range of the scale parameter of the basis. The matrix elements are then compared with the parameterizations given above to determine the semileptonic transition form factors.

\section{Semileptonic $B$ decays}

In Fig. 3 we give the form factors relevant for the transition of $B \rightarrow D$ and $B \rightarrow D^{*}$. They are calculated using the Salpeter amplitudes reproducing the meson mass spectra as shown in Fig. 1. Our calculation is given by the solid line. The mono-pole dominance ansatz of Bauer, Stech and Wirbel (BSW) [47] is shown as a long dashed line. The short dashed line shows an earlier result within the non-relativistic framework (CQM). The latter, however, includes relativistic corrections in the current operators [16].

In Table 2 we explicitly give the ratios of the form factors $A_{2} / A_{1}$ and $V / A_{1}$ at $q^{2}=q_{\max }^{2}$. The values fit experimental data, which however, have rather large error bars. For comparison we have also given other model values as compiled in [32], in particular of the improved non-relativistic constituent quark model given earlier [16].

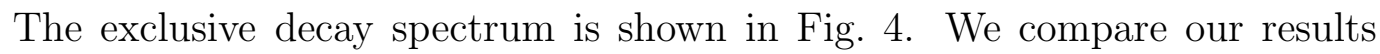
to recent ARGUS [35] and CLEO data [37, 38. We find a best fit with $V_{c b}=$ $(0.032 \pm 0.003)\left(\tau_{B} / 1.49 p s\right)^{1 / 2}$. The error resulting from $\chi^{2}$ fitting is indicated by the upper and lower dotted line. Our previous result using a non-relativistic formalism with relativistic corrections in the current operators is given by the dashed line. We find $V_{c b}$ consistent with each other [16].

The resulting total branching ratios for semileptonic $B$ decays agree well with experimental data given by the Particle Data Group [32], and by the CLEO collaboration [38] and ARGUS collaboration [35]. They are given in Table 3.

In Table 1 we give the resulting forward backward asymmetry $A_{F B}$ and the asymmetry parameter $\alpha$ defined e.g. in [36] respecting the lepton cut-off momentum of the CLEO collaboration. The forward backward asymmetry sensitive to 
parity violation is defined through

$$
A_{F B}=\frac{N_{F}-N_{B}}{N_{F}+N_{B}}
$$

with $N$ the number of leptons in forward, resp. backward hemisphere in the rest system of the $W$-boson. For $A_{F B}$ we have used a symmetric cut on the lepton momentum $p_{\ell}$ as utilized by the CLEO collaboration [38] for technical reasons.

The helicity alignment $\alpha$ describes the $D^{*+}$ polarization extracted from the $D^{*+} \rightarrow D^{0} \pi^{+}$decay angle distribution $W\left(\theta^{*}\right)$, viz.

$$
W\left(\theta^{*}\right) \propto 1+\alpha \cos ^{2} \theta^{*}
$$

For $\alpha$ only a lower cut has been introduced. Both observables are in good agreement with experimental results.

We would now like to connect our results to the notion of heavy quark symmetry. In this context we reparametrize the form factors in terms of $h_{+}(\omega), h_{-}(\omega)$ for $0^{-} \rightarrow 0^{-}$, and $h_{V}(\omega), h_{A_{1}}(\omega), h_{A_{2}}(\omega), h_{A_{3}}(\omega)$ for $0^{-} \rightarrow 1^{-}$transitions, with

$$
\omega=\frac{m_{B}^{2}+m_{D^{(*)}}^{2}-q^{2}}{2 m_{B} m_{D^{(*)}}}
$$

The transformation equations are given in [16]. The advantage of this parameterization is that in the heavy quark mass limit $\left(m_{c, b} \rightarrow \infty\right)$

$$
\begin{aligned}
h_{V}(\omega)=h_{A_{1}}(\omega)=h_{A_{3}}(\omega) & =h_{+}(\omega)=\xi(\omega) \\
h_{A_{2}}(\omega) & =h_{-}(\omega)=0
\end{aligned}
$$

where $\xi(\omega)$ is a universal function known as Isgur Wise function [28]. The heavy quark mass limit has been performed numerically by multiplying $m_{c, b}$ with a large factor, while keeping all other parameters as given in Table 1. The meson amplitudes to evaluate the transition matrix elements (14) are then calculated by diagonalizing the equivalent eigenvalue problem. Due to numerical reasons the heavy quark masses cannot be chosen too large. However, a suitable choice leads to converging form factors of eq. (21), which differ by less than $0.1 \%$. This resulting function is then defined to be the Isgur Wise function $\xi_{I B S}(\omega)$. Its values are shown in Table 5 .

We find that is it possible to approximately parameterize this function by either a pole or an exponential fit, viz.

$$
\begin{aligned}
\xi_{\text {pole }}(\omega) & \simeq \frac{\Lambda^{2}+1}{\Lambda^{2}+\omega} \\
\xi_{\text {exp }}(\omega) & \simeq \exp \left[\alpha^{2}(1-\omega)\right]
\end{aligned}
$$


Choosing $\Lambda=0.14$, or $\alpha^{2}=0.80$ the deviation of this fits from the calculated values $\xi_{I B S}(\omega)$ are smaller or about $1 \%$, whereby $\xi_{\text {exp }}(\omega)$ leads to a slightly better overall fit.

In Figure 5 we show the deviations of the IBS form factors, $h_{V}(\omega), h_{A_{1}}(\omega)$, $h_{A_{3}}(\omega), h_{+}(\omega)$, (viz. without implementing the 'numerical' heavy quark mass limit) from the ideal heavy quark limit form factor $\xi(\omega)$. The form factor $h_{V}(\omega)$ deviates rather strongly from the Isgur Wise function, whereas $h_{+}$and $h_{A_{3}}$ are within $10 \%$. Although our result is not a $\Lambda_{Q C D} / m_{q}$ expansion, it is interesting to note, that $h_{A_{1}}(1)=0.995$ agrees with the expectation of Luke's theorem [42], and $h_{A_{1}}(\omega)$ is rather close to the limiting case $\xi_{I B S}(\omega)$.

\section{Semileptonic $D$ decays}

In Figure 6 we show the $D \rightarrow K^{(*)}$ form factors. The results of the IBS model are compared to other models as well as to empirical data. The empirical data points at $q^{2}=0$ are given by the particle data group assuming a pole dominance behaviour of the form factors [32]. Note that the covariant treatment (solid line) can reproduce a pole-like behavior in contrast to the non-relativistic calculations (dashed line).

Except for the form factor $A_{1}(0)$ the model form factors agree reasonably well with the empirical values given. It is remarkable that $A_{2}(0)$ turns out to be much closer to the empirical value than the non-relativistic result or the BSW values that are fixed by quark wave functions in the infinite momentum frame. On the other hand $A_{1}(0)$ is too large by $50 \%$.

In Table 6 we show the resulting partial decay rates of $D \rightarrow K^{(*)}, D_{s} \rightarrow \Phi$. Although the decay into $K$ reproduces the experimental value nicely, the decays into $K^{*}$ and $\Phi$ are rather off the experimental data. In addition the ratio of helicity rates $\Gamma_{+} / \Gamma_{-}$shown in Table 7 turns out larger than the experimental ones. This reflects the difference in $A_{1}(0)$ between the experimental and empirical values. Table 7 also shows the ratio of longitudinal to transverse polarized rates $\left(\Gamma_{L} / \Gamma_{T}=\Gamma_{0} /\left(\Gamma_{-}+\Gamma_{+}\right)\right)$, which is well reproduced by the model.

The decay into $\eta$ is not calculated since this requires a discussion of $\eta, \eta^{\prime}$ mixing which is not touched here. At the present stage it would require additional assumptions and the introduction of mixing parameters, which we like to exclude here. Such mixing may be generated in a natural way though instanton effects [45], or two gluon exchanges. The consequences of instanton effects have been studied in 18, 19. 


\section{Non-leptonic decays}

Due to strong interaction non-leptonic weak decays provide additional phenomena. Examples are hard gluon exchanges, quark rearrangement, annihilation and long range effects. Thus, extraction of fundamental physical constants such as the Cabbibo-Kobayashi-Maskawa matrix elements is more difficult than in the semileptonic case. Discussion on QCD corrections have been triggered by the recent CLEO data on $B$ meson decays. The rôle of QCD correction needs clarification, however, such a discussion goes beyond the scope of the present paper. For definiteness we shall neglect all gluonic effects in the weak operators. Implicitly some gluonic effects are included in the Bethe Salpeter amplitudes via the interaction kernel. Therefore we also need not to worry about double counting etc. The simple weak interaction kernel for non-leptonic decay may then be written in current approximation as

$$
\mathcal{L}_{e f f}=-\frac{G_{F}}{\sqrt{2}} \sum_{\alpha^{\prime} \beta^{\prime} \alpha \beta} V_{\alpha^{\prime} \beta^{\prime}} V_{\alpha \beta}^{*} h_{\mu, \alpha \beta} h_{\alpha^{\prime} \beta^{\prime}}^{\mu}
$$

with $\alpha, \alpha^{\prime} \in\{u, c\}, \beta, \beta^{\prime} \in\{d, s\}$. The Lagrangian (25) is then evaluated between the meson amplitudes in the same way as for semileptonic decays, given in (14).

Note that due to Fierz rearrangement two generic types of contributions are possible. These are demonstrated in Figure 7 . The generic form of class I is given by the following eq. (26) those of class II by eq. (27), class III are mixed forms of both.

$$
\begin{aligned}
\left\langle\pi^{+} D^{-}\left|h_{\mu, u d} h_{c b}^{\mu}\right| B^{0}\right\rangle & \rightarrow\left\langle\pi^{+}\left|h_{\mu, u d}\right| 0\right\rangle\left\langle D^{-}\left|h_{c b}^{\mu}\right| B^{0}\right\rangle \\
\left\langle\pi^{0} D^{0}\left|h_{\mu, u d} h_{c b}^{\mu}\right| B^{0}\right\rangle & \rightarrow \frac{1}{3}\left\langle D^{0}\left|h_{\mu, c d}\right| 0\right\rangle\left\langle\pi^{0}\left|h_{u b}^{\mu}\right| B^{0}\right\rangle
\end{aligned}
$$

The factor $1 / 3$ stems from the colour suppression of the Fierz rearranged diagram.

In order to separate the physical effects, we have used empirical values for the vacuum transition part $\left\langle X\left|h_{\mu}\right| 0\right\rangle$ to evaluate the non-leptonic transition matrix elements. The empirical values for the decay constants used in the calculation of non-leptonic decays along with the calculated ones from the IBS model are shown

in Table 8. They are not so well reproduced by the IBS model, however calculated values are by orders of magnitudes closer to the empirical ones compared to the non-relativistic approach.

Experimental values of decay constants are available only for $\pi$ and $K$ from leptonic weak decays. For the $D$-meson decay constant only an upper limit exists, $f_{D^{+}}<310 \mathrm{MeV}$. For $f_{D_{s}}$ we have chosen $f_{D_{s}}=300 \mathrm{MeV}$, which is close to values found by Rosner [40] and an ARGUS analysis using different types of model analyses [41]. For $f_{\rho}$ we have used $f_{\rho}=205 \mathrm{MeV}$, which has been suggested by [44]. Other decay constants are taken from [46]. 
We consider only those non-leptonic decays where experimental values are known. These are taken from the compilation of the Particle Data Group [32], which include also some (preliminary) results from recent CLEO [38] and ARGUS [41, 35] collaborations.

Results are shown in Tables 9 to 13. The Cabbibo Kobayashi Maskawa matrix elements used and $V_{c b}=0.032$ and others are given in 32 . The description of $B$ meson non-leptonic decays is rather satisfactory. The relative sign between the amplitudes of type III decays consistent with the Lagrangian given in eq. (25) is positive. This holds also for $D$ meson decays. On the other hand, experimental values for type III $D$ decays are not reproduced. This is usually attributed to gluonic effects. However, we shall not elaborate on this point any further.

\section{Summary and Conclusion}

Utilizing the Bethe Salpeter equation we have calculated the meson mass spectrum, as well as weak semileptonic decay observables of $B$ and $D$ mesons. The interaction kernel has been assumed instantaneous. This way the Bethe Salpeter equation reduces to a (full) Salpeter equation as given in eq.(1). The interaction consists of a one gluon exchange evaluated in the Coulomb gauge and a linear confinement, which are given in co-ordinate space. The parameters of the model are fixed to reproduce the meson mass spectrum.

To evaluate current matrix elements, and in order to make explicit covariance transparent, the Bethe Salpeter amplitude $\chi_{P}\left(p^{0}, \boldsymbol{p}\right)$ has been fully reconstructed. In this way it is possible to extract form factors by comparison of the Lorentz structure of the standard notation of the current.

To calculate the Isgur Wise function the heavy quark mass limit has been achieved in a numerical sense. Within the model uncertainties the resulting function may be parameterized by an exponential as well as by a pole-like function. Deviation of the form factor $h_{A_{1}}(0)$ from unity is very small as expected from Luke's theorem. Although it is possible to extract the value of the Cabbibo Kobayashi Maskawa matrix element $V_{c b}$ without knowing the exact shape of the Isgur Wise function, its notion is desirable since it leads to more insight into the dynamical mechanism of the $q \bar{q}$ system.

From the semileptonic decay rate $B \rightarrow D^{*} e \nu$ the Cabbibo Kobayashi Maskawa matrix element turns out to be $V_{c b}=0.032 \pm 0.003$, which is close to the nonrelativistic value. We have taken $\tau_{B}=1.49 \mathrm{ps}$ [32] for the $B$ meson life time.

As a main outcome of the covariant treatment we find, that the empirical pole behavior of the form factors of the semileptonic $D$ decays is well reproduced. We consider this as an important advantage with respect to the non-relativistic 
treatment, where this is not possible. Also empirical values are reasonably well reproduced except for $A_{1}\left(q^{2}\right)$. The total decay rate for $D \rightarrow K e \nu$ is reproduced rather well, whereas for $D \rightarrow K^{*} e \nu$ and $D_{s} \rightarrow \phi e \nu$ experimental values are overestimated. Since presently no model appears able to explain all form factors, this may well have a more profound reason than a simple shortcoming of the model. Changing the model parameters given in Table [ may lead to some improvement on $A_{1}\left(q^{2}\right)$ however, the mesonic mass spectrum Figure 11 cannot be reproduced then.

As a further application we have calculated the non-leptonic decays leaving the discussion of gluonic corrections and final state interaction aside. We find reasonable results, however without solving the relative sign problem of type III decays. This needs further consideration, which is beyond our present intention.

In conclusion, we presented a covariant model to treat mesons in terms of the underlying quark fields, respecting simple QCD phenomena such as confinement and spin splitting. It allows to extract fundamental quantities of the standard model from hadronic degrees of freedom in a consistent way using Mandelstam formalism. Since the model leads to reasonable results one may now also address more exotic phenomena, such as penguin diagrams or rare decays.

\section{Acknowledgment}

One of us (M.B.) is grateful to Yura Kalinovsky for reading the manuscript and a valuable discussion on the Isgur Wise function. This work was supported in part by the Bundesminister für Forschung und Technologie. 
Table 1: Parameter of the model kernel

\begin{tabular}{cccccccc}
\hline \hline $\begin{array}{c}m_{u, d} \\
{[\mathrm{GeV}]}\end{array}$ & $\begin{array}{c}m_{s} \\
{[\mathrm{GeV}]}\end{array}$ & $\begin{array}{c}m_{c} \\
{[\mathrm{GeV}]}\end{array}$ & $\begin{array}{c}m_{b} \\
{[\mathrm{GeV}]}\end{array}$ & $\begin{array}{c}a_{c} \\
{[\mathrm{GeV}]}\end{array}$ & $\begin{array}{c}b_{c} \\
{[\mathrm{GeV} / \mathrm{fm}]}\end{array}$ & $\begin{array}{c}r_{0} \\
{[\mathrm{fm}]}\end{array}$ & $\alpha_{\text {sat }}$ \\
\hline 0.200 & 0.440 & 1.738 & 5.110 & -1.027 & 1.700 & 0.1 & 0.391 \\
\hline \hline
\end{tabular}

Table 2: Comparison of ratios of form factors for $B \rightarrow \bar{D}^{*} \ell^{+} \nu_{\ell}$ at $q^{2}=q_{\max }^{2}$

\begin{tabular}{lcc}
\hline \hline & $\left(A_{2} / A_{1}\right)\left(q_{\max }^{2}\right)$ & $\left(V / A_{1}\right)\left(q_{\max }^{2}\right)$ \\
\hline CLEOII fit (a) & $1.02 \pm 0.24$ & $1.07 \pm 0.57$ \\
CLEOII fit (b) & $0.79 \pm 0.28$ & $1.32 \pm 0.62$ \\
IBS & 1.10 & 1.41 \\
CQM & 1.42 & 1.15 \\
BSW & 1.06 & 1.14 \\
KS & 1.39 & 1.54 \\
\hline \hline
\end{tabular}

Table 3: Branching ratios for $\mathrm{B}$ decays. We use $\tau_{B}=1.49 \mathrm{ps}$, a) recent CLEO, b) recent ARGUS data

\begin{tabular}{ccc}
\hline \hline decay & $B r_{I B S}[\%]$ & $B r_{\text {exp }}[\%]$ \\
\hline $\bar{B}^{0} \rightarrow D^{*+} \ell^{-} \bar{\nu}_{\ell}$ & 4.2 & $4.9 \pm 0.8$ \\
& & $4.50 \pm 0.44 \pm 0.44^{a}$ \\
& & $5.2 \pm 0.5 \pm 0.6^{b}$ \\
$\bar{B}^{0} \rightarrow D^{+} \ell^{-} \bar{\nu}_{\ell}$ & 1.6 & $1.6 \pm 0.7$ \\
\hline \hline
\end{tabular}


Table 4: Forward backward asymmetry and asymmetry parameter $\alpha$ for the decay $\bar{B}^{0} \rightarrow D^{*+} \ell^{-} \bar{\nu}_{\ell}$. CLEO with lepton cut-off $p_{\ell}^{\text {cut }}=1.0 / 1.4 \mathrm{GeV}$, ARGUS without cut-off

\begin{tabular}{cccc}
\hline \hline & & & \\
& $p_{\ell}^{c u t}$ & IBS & experiment \\
\hline & & & \\
$A_{F B}$ & 0 & 0.20 & $0.2 \pm 0.1$ \\
& 1.0 & 0.14 & $0.14 \pm 0.07$ \\
$\alpha$ & 0 & 1.37 & $1.1 \pm 0.4$ \\
& 1.4 & 0.50 & $0.65 \pm 0.7$ \\
\hline \hline
\end{tabular}

Table 5: Isgur Wise function calculated from the instantaeous Bethe Salpeter equation as explained in the text

\begin{tabular}{cc}
\hline \hline$\omega$ & $\xi(\omega)$ \\
\hline 1.00 & 1.000 \\
1.05 & 0.958 \\
1.10 & 0.918 \\
1.15 & 0.880 \\
1.20 & 0.845 \\
1.25 & 0.811 \\
1.30 & 0.780 \\
1.35 & 0.750 \\
1.40 & 0.721 \\
1.45 & 0.695 \\
1.50 & 0.669 \\
1.55 & 0.645 \\
1.60 & 0.623 \\
\hline \hline
\end{tabular}

Table 6: $D \rightarrow K^{(*)}$ and $D_{s} \rightarrow \phi$ decays

\begin{tabular}{ccc}
\hline \hline decay mode & $\Gamma_{\mathrm{IBS}}\left[10^{10} s^{-1}\right]$ & $\Gamma_{\exp }\left[10^{10} s^{-1}\right]$ \\
\hline & & \\
$D \rightarrow \bar{K} e \nu_{e}$ & 8.8 & $8.2 \pm 0.4$ \\
$D \rightarrow \bar{K}^{*} e \nu_{e}$ & 8.2 & $4.6 \pm 0.4$ \\
\hline$D_{s} \rightarrow \phi \ell^{+} \bar{\nu}_{\ell}$ & 7.7 & $3.11 \pm 1.11$ \\
\hline \hline
\end{tabular}


Table $7: D \rightarrow K^{(*)}$ helicity ratios

\begin{tabular}{lcc}
\hline \hline & $\Gamma_{L} / \Gamma_{T}$ & $\Gamma_{+} / \Gamma_{-}$ \\
\hline & & \\
exp. average & $1.23 \pm 0.13$ & $0.16 \pm 0.04$ \\
IBS & 1.38 & 0.30 \\
\hline
\end{tabular}

Table 8: Empirical decay constants $f_{\text {emp }}$ used for calculating nonleptonic decays compared to calculated IBS values $f_{I B S}$

\begin{tabular}{ccc}
\hline \hline & & \\
meson & $f_{\text {emp }}$ & $f_{I B S}$ \\
type & {$[\mathrm{MeV}]$} & {$[\mathrm{MeV}]$} \\
\hline & & \\
$\pi^{+}$ & 132 & 153 \\
$\pi^{0}$ & 93 & 108 \\
$K^{ \pm}$ & 162 & 220 \\
$\bar{K}^{0}$ & 162 & 220 \\
$D^{+}$ & 220 & 293 \\
$D^{0}$ & 220 & 293 \\
$D_{s}^{+}$ & 300 & 342 \\
$\rho^{+}$ & 205 & 480 \\
$\rho^{0}$ & 145 & 339 \\
$K^{*-}$ & 220 & 503 \\
$\bar{K}^{* 0}$ & 220 & 503 \\
$D^{*+}$ & 220 & 409 \\
$D^{* 0}$ & 220 & 409 \\
$D_{s}^{*+}$ & 300 & 467 \\
$J / \Psi$ & 382 & 571 \\
$a_{1}^{+}$ & 220 & 371 \\
\hline \hline
\end{tabular}


Table 9: Decay widths of weak nonleptonic $B^{0}$ decays in units of $s^{-1}$. Upper part type I, lower type II decays

\begin{tabular}{lll}
\hline \hline & & \\
$B^{0}$ decays & $\Gamma_{I B S}$ & $\Gamma_{\text {exp }}$ \\
\hline & & \\
$D^{-} \pi^{+}$ & 0.208 & $0.20 \pm 0.03$ \\
$D^{-} \rho^{+}$ & 0.465 & $0.52 \pm 0.09$ \\
$D^{-} D_{s}^{+}$ & 0.840 & $0.53 \pm 0.27$ \\
$D^{*-} D_{s}^{+}$ & 0.667 & $0.80 \pm 0.40$ \\
$D^{-} D_{s}^{*+}$ & 0.451 & $1.40 \pm 1.0$ \\
$D^{*-} D_{s}^{*+}$ & 0.914 & $1.33 \pm 0.80$ \\
$D^{*-} \rho^{+}$ & 0.522 & $0.49 \pm 0.10$ \\
\hline$K^{0} J / \Psi$ & 0.033 & $0.05 \pm 0.01$ \\
$K^{* 0} J / \Psi$ & 0.075 & $0.11 \pm 0.02$ \\
\hline \hline
\end{tabular}

Table 10: Decay widths of weak nonleptonic $B^{+}$decays in units of $s^{-1}$. From upper to lower part: type I, II, III decays, resp.

\begin{tabular}{lll}
\hline \hline & & \\
$B^{+}$decays & $\Gamma_{I B S}$ & $\Gamma_{\text {exp }}$ \\
\hline $\bar{D}^{0} D_{s}^{+}$ & 0.840 & $1.10 \pm 0.39$ \\
$\bar{D}^{0} D_{s}^{*+}$ & 0.451 & $0.78 \pm 0.65$ \\
$\bar{D}^{* 0} D_{s}^{+}$ & 0.667 & $0.65 \pm 0.45$ \\
$\bar{D}^{* 0} D_{s}^{*+}$ & 0.914 & $1.56 \pm 0.84$ \\
\hline$K^{+} J / \Psi$ & 0.033 & $0.07 \pm 0.01$ \\
$K^{*+} J / \Psi$ & 0.075 & $0.11 \pm 0.03$ \\
\hline $\bar{D}^{0} \pi^{+}$ & 0.217 & $0.34 \pm 0.03$ \\
$\bar{D}^{0} \rho^{+}$ & 0.507 & $0.87 \pm 0.12$ \\
$\bar{D}^{0 *} \pi^{+}$ & 0.307 & $0.34 \pm 0.05$ \\
$\bar{D}^{0 *} \rho^{+}$ & 0.561 & $1.01 \pm 0.20$ \\
\hline \hline
\end{tabular}


Table 11: Decay widths of weak nonleptonic $D^{0}$ decays in units of $s^{-1}$. Upper part type I, lower part type II decays.

\begin{tabular}{lll}
\hline \hline & & \\
$D^{0}$ decays & $\Gamma_{I B S}$ & $\Gamma_{\text {exp }}$ \\
\hline$K^{-} \pi^{+}$ & 10.52 & $9.66 \pm 0.34$ \\
$K^{-} \rho^{+}$ & 15.79 & $25.06 \pm 3.13$ \\
$\bar{K}^{0} \phi$ & 0.58 & $2.00 \pm 0.29$ \\
$K^{-} a_{1}^{+}$ & 2.21 & $19.04 \pm 2.89$ \\
$K^{*-} \pi^{+}$ & 7.87 & $11.81 \pm 1.45$ \\
$K^{* 0} \pi^{0}$ & 0.002 & $7.23 \pm 0.96$ \\
$K^{*-} \rho^{+}$ & 11.29 & $14.22 \pm 5.78$ \\
$\pi^{+} \pi^{-}$ & 0.14 & $0.38 \pm 0.03$ \\
$K^{+} K^{-}$ & 0.78 & $1.09 \pm 0.07$ \\
$K^{0} \bar{K}^{0}$ & 0.78 & $0.27 \pm 0.10$ \\
$K^{*+} K^{-}$ & 0.43 & $0.82 \pm 0.19$ \\
$K^{*-} K^{+}$ & 0.43 & $0.43 \pm 0.24$ \\
$K^{* 0} \bar{K}^{* 0}$ & 0.40 & $0.70 \pm 0.39$ \\
\hline$K^{0} \pi^{0}$ & 0.001 & $4.94 \pm 0.63$ \\
$\bar{K}^{0} \rho^{0}$ & 3.00 & $2.65 \pm 0.43$ \\
$\pi^{0} \pi^{0}$ & 0.07 & $0.21 \pm 0.06$ \\
$\phi \rho^{0}$ & 0.04 & $0.46 \pm 0.12$ \\
\hline \hline
\end{tabular}

Table 12: Decay widths of weak nonleptonic $D_{(s)}^{+}$decays in units of $s^{-1}$. Upper part type I, lower type III decays

\begin{tabular}{lll}
\hline \hline & & \\
$D^{+}$decay & $\Gamma_{I B S}$ & $\Gamma_{\text {exp }}$ \\
\hline $\bar{K}^{0} a_{1}^{+}$ & 2.21 & $7.66 \pm 1.61$ \\
$\bar{K}^{0} K^{+}$ & 0.78 & $0.74 \pm 0.16$ \\
$\phi \pi^{+}$ & 0.031 & $0.63 \pm 0.08$ \\
$\bar{K}^{* 0} K^{+}$ & 0.43 & $0.48 \pm 0.09$ \\
$K^{*+} \bar{K}^{* 0}$ & 0.40 & $2.46 \pm 1.04$ \\
\hline$K^{0} \pi^{+}$ & 11.25 & $2.59 \pm 0.27$ \\
$\bar{K}^{0} \rho^{+}$ & 17.11 & $6.24 \pm 2.37$ \\
$\bar{K}^{* 0} \pi^{+}$ & 7.91 & $2.08 \pm 0.38$ \\
$\pi^{+} \pi^{0}$ & 2.07 & $0.24 \pm 0.07$ \\
\hline \hline
\end{tabular}


Table 13: Decay widths of weak nonleptonic $D_{(s)}^{+}$decays in units of $s^{-1}$. Upper part type I lower part type II decays

\begin{tabular}{lll}
\hline \hline & & \\
$D_{s}^{+}$decay & $\Gamma_{I B S}$ & $\Gamma_{\text {exp }}$ \\
\hline$K^{+} \bar{K}^{* 0}$ & 1.45 & $7.49 \pm 1.50$ \\
$\phi \pi^{+}$ & 7.35 & $7.49 \pm 0.86$ \\
$\phi \rho^{+}$ & 10.68 & $13.92 \pm 3.43$ \\
\hline$K^{+} \bar{K}^{* 0}$ & 1.76 & $7.07 \pm 1.07$ \\
$K^{*+} \bar{K}^{0}$ & 0.93 & $8.99 \pm 2.14$ \\
\hline \hline
\end{tabular}



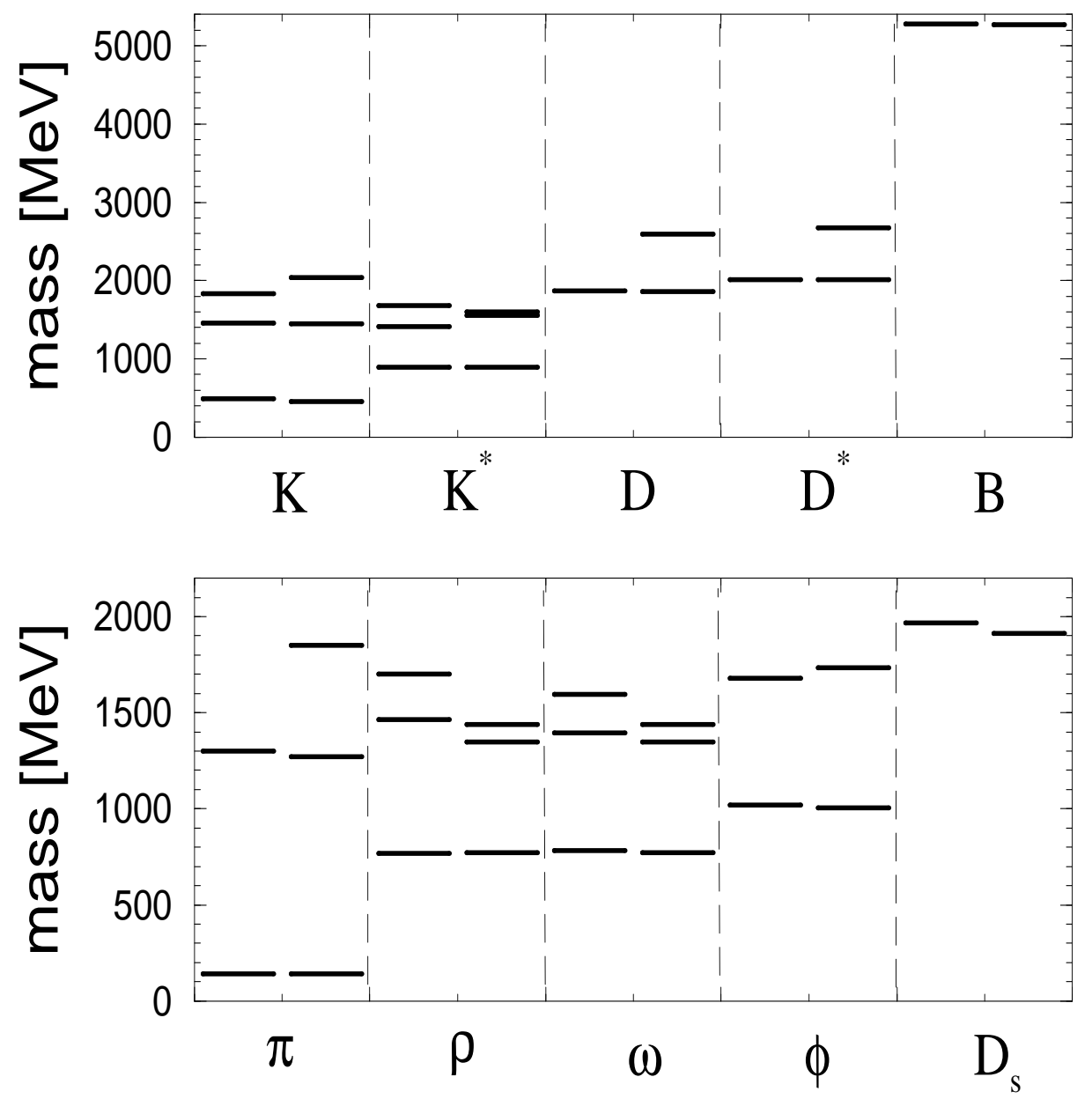

Figure 1: Meson mass spectrum for low radial excitation. Left column experimental, right column model results, resp. 


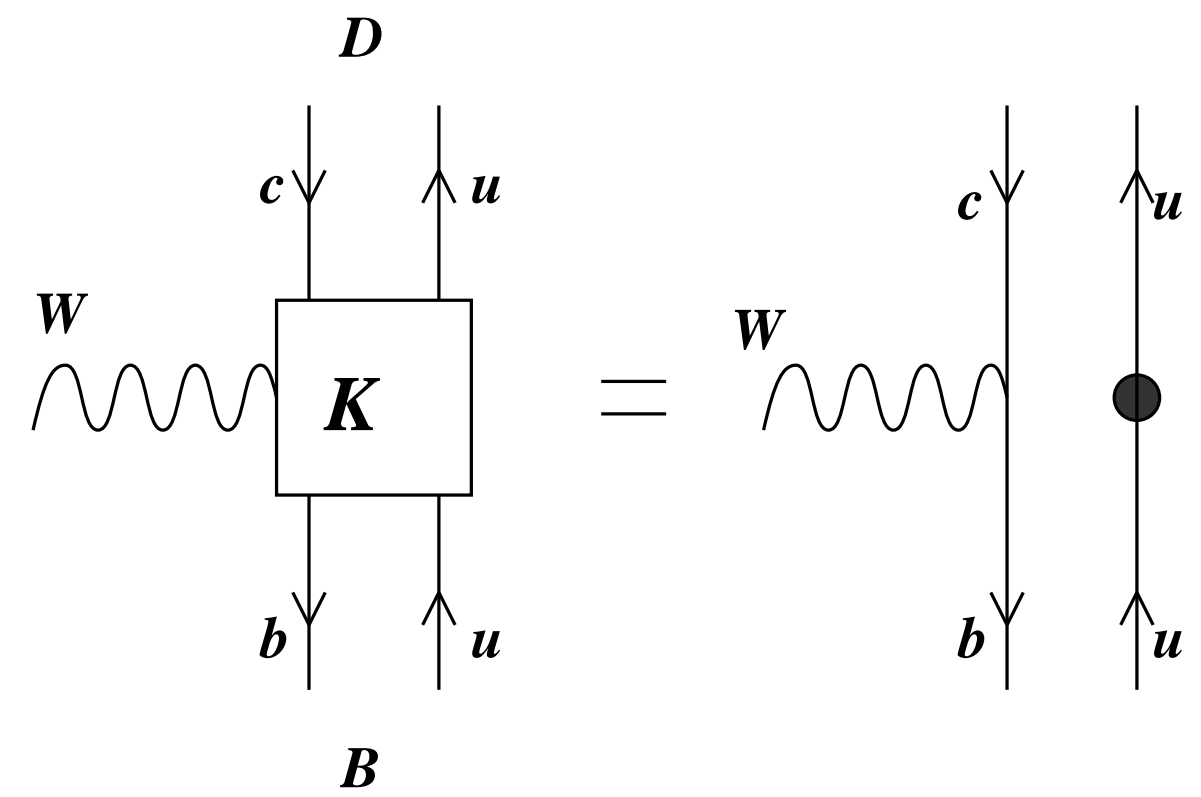

Figure 2: Pictorial demonstration of one particle approximation of the irreducible interaction kernel eq.(13), filled circle denotes the inverse quark propagator 

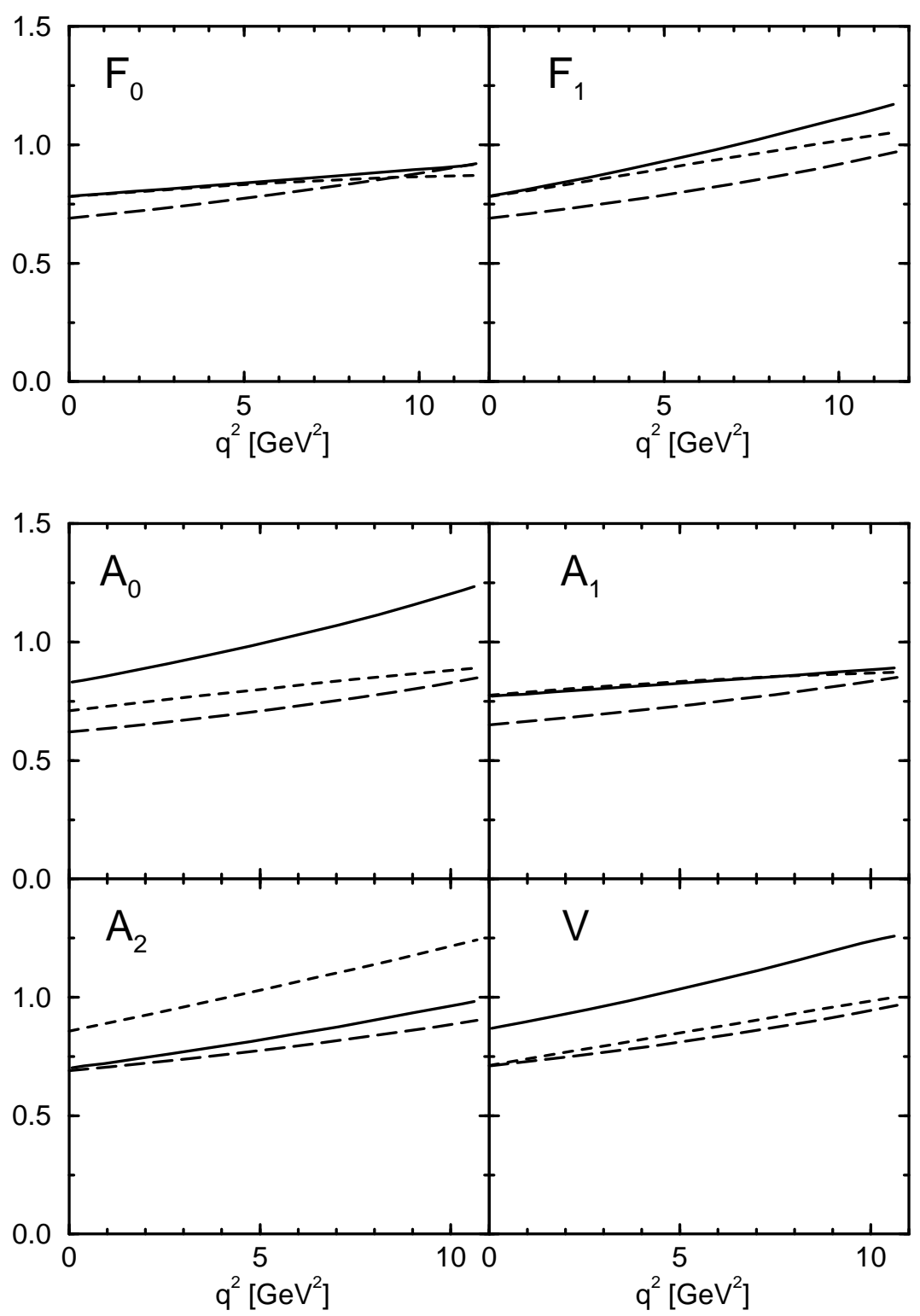

Figure 3: A comparison of form factors for $\mathrm{B} \rightarrow \mathrm{D}$ and $\mathrm{B} \rightarrow D^{*}$ transitions; our result (solid line), non relativistic result with relativistic corrections (dashed), BSW (long-dashed) 


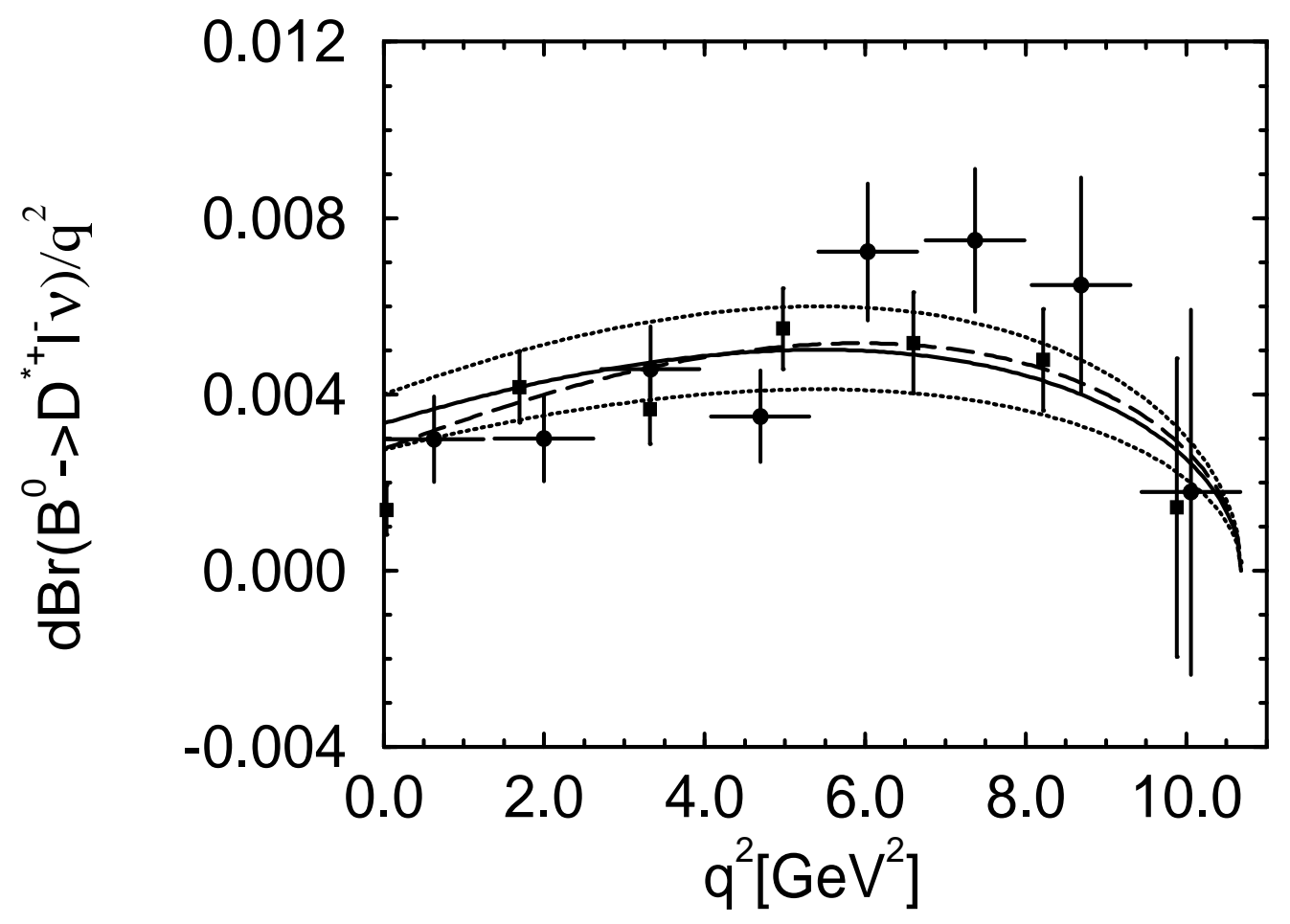

Figure 4: $q^{2}$ distribution of $B \rightarrow D^{*+} \ell^{-} \bar{\nu}$. Experiments given by ARGUS (circles) and CLEO (squares). The solid line calculated with $V_{c b}=0.032$ and life time $\tau_{B}=1.49 \mathrm{ps}$; the upper and lower dotted lines with $V_{c b}=0.032 \pm 0.003$ respectively. The dashed line corresponds to the nonrelativistic treatment with $V_{c b}=0.034 \pm 0.003$. 


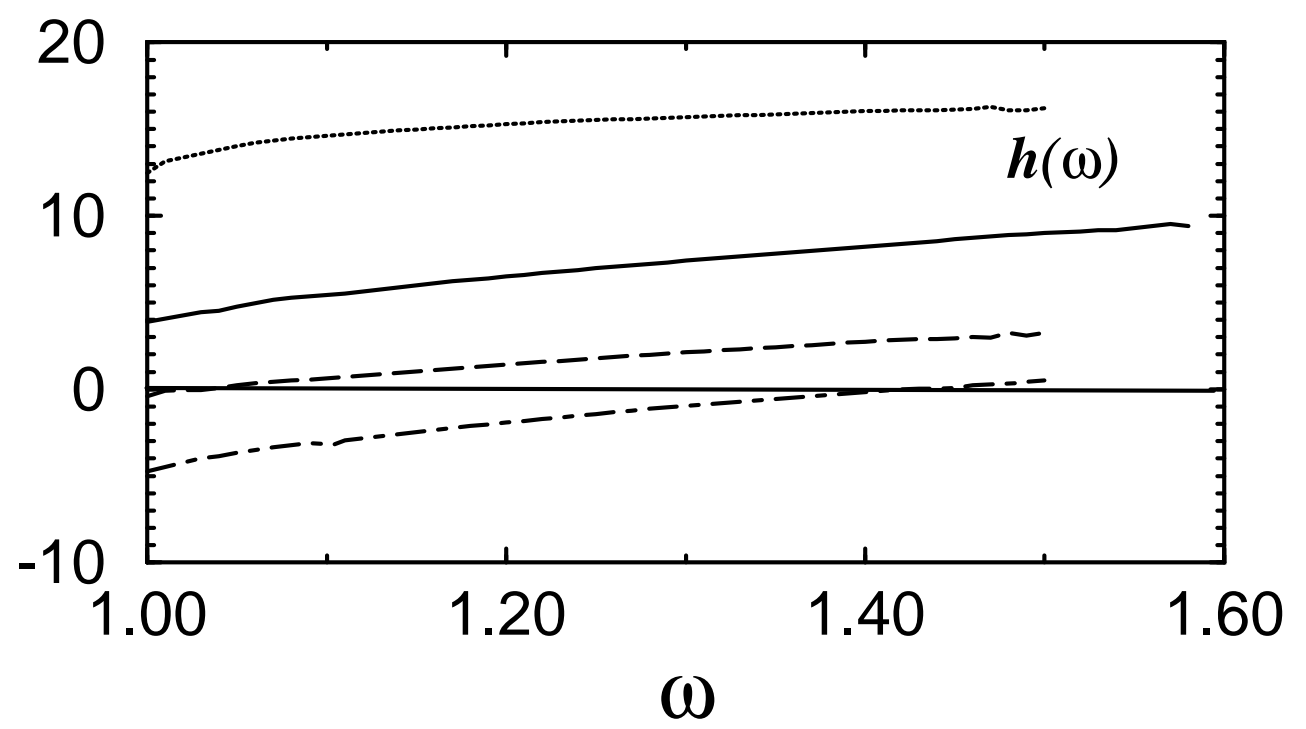

Figure 5: Deviation of the model form factors from the Isgur Wise function in [\%]. From top to bottom: $h_{V}, h_{+}, h_{A_{1}}, h_{A_{3}}$. 

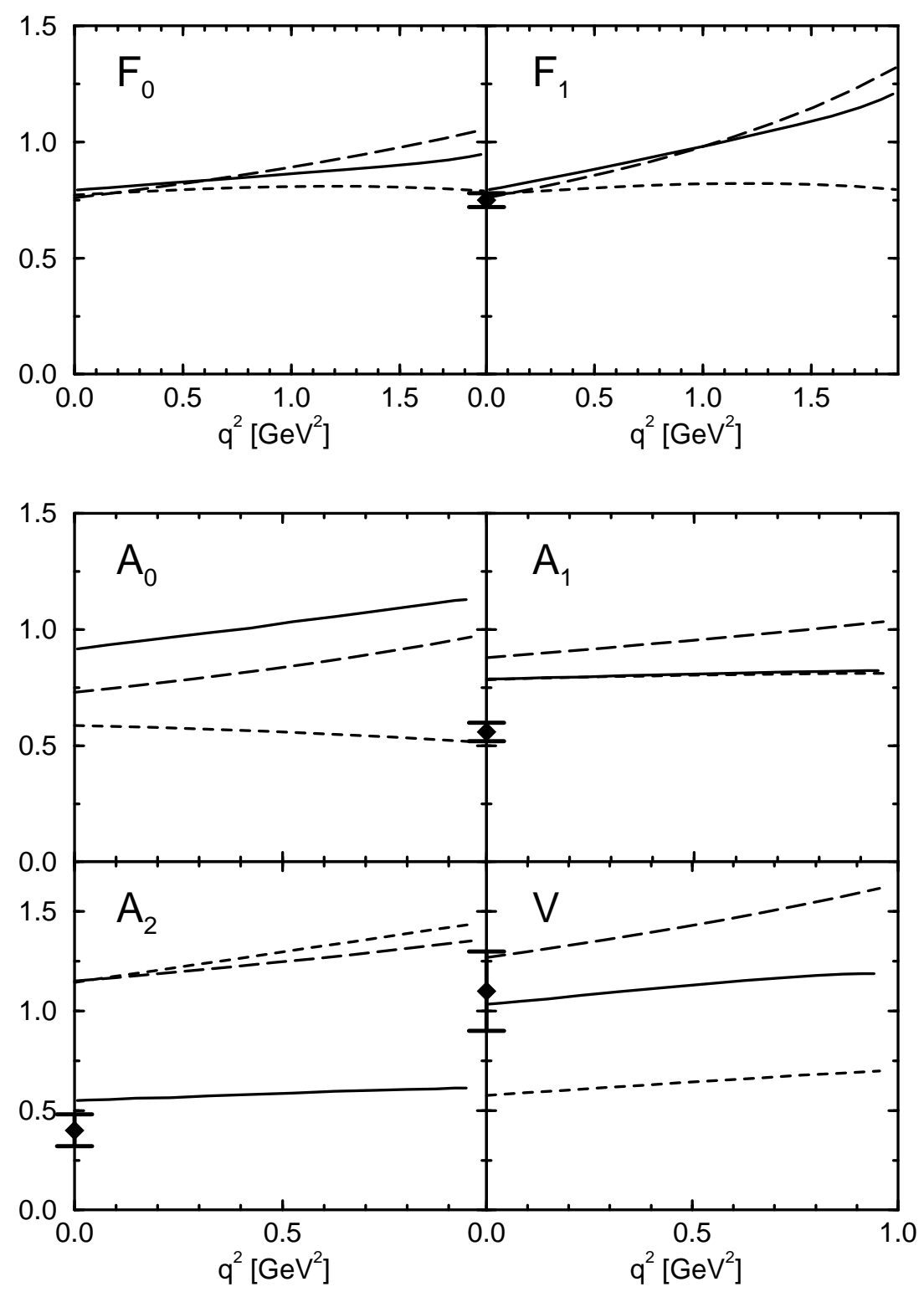

Figure 6: A comparison of form factors for $\mathrm{D} \rightarrow \mathrm{K}$ and $\mathrm{D} \rightarrow K^{*}$ transitions; our result (solid line), non relativistic result with relativistic corrections (dashed), BSW (long-dashed), empirical form factors (shaded area, where given) 

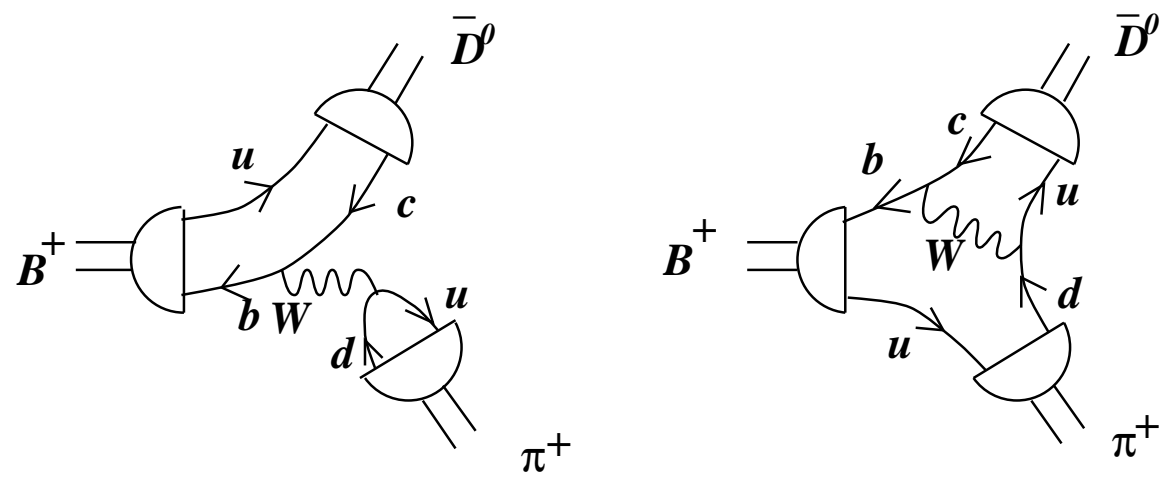

Figure 7: Type I (left) and Type II (right) nonleptonic decays. In the limit of heavy W-Boson, Type II decays can be Fierz transformed. 


\section{References}

[1] A. De Rújula, H. Georgi, S.L. Glashow: Phys. Rev. D 12 (1975) 147

[2] E. Eichten et al.: Phys. Rev. D 17 (1978) 3090; D 21 (1980) 203

[3] C. Quigg, J.L. Rosner: Phys. Lett. 21B (1977) 252; W. Kwong, J.L. Rosner, C. Quigg: Ann. Rev. Nucl. Part. Sci. 37 (1987) 325

[4] W. Celmaster, H. Georgi, M. Machazek: Phys. Rev. D17 (1978) 879

[5] G. Bhanot, S. Rudaz: Phys. Lett. 78B (1978) 119

[6] J. Richardson: Phys. Lett. 82B (1979) 272

[7] G. Fogleman, D.B. Lichtenberg, J.G. Wills: Lett. Nuovo Cim. 26 (1979) 369

[8] W. Buchmüller, S.-H. Tye: Phys. Rev. D 24 (1981) 132

[9] A. Martin: Phys. Lett. 93B (1980) 338; 100B (1981) 511

[10] X.T. Song, H. Lin: Z. Phys. C - Particles and Fields 34 (1987) 223

[11] D.B. Lichtenberg et al.: Z. Phys. C - Particles and Fields 41 (1989) 615

[12] D.B. Lichtenberg, R. Roncaglia, L.G. Wills: Z. Phys. C - Particles and Fields 46 (1990) 75

[13] D.B. Lichtenberg: Int. J. Mod. Phys. A2 (1987) 1669

[14] W. Lucha, F.F. Schöberl, D. Gromes: Phys. Rep. 200 (1991) 127

[15] M. Beyer, U. Bohn, M.G. Huber, B.C. Metsch, J. Resag, Z. Phys. C - Particles and Fields 55 (1992) 307

[16] S. Resag, M. Beyer: Z. Phys. 63 (1994) 121

[17] S. Resag: diploma thesis (unpublished) BONN TK-93-17

[18] J. Resag, C.R. Münz, B.C. Metsch, H.R. Petry: Nucl. Phys. A (1994) 397

[19] C.R. Münz, J. Resag, B.C. Metsch, H.R. Petry: Nucl. Phys. A (1994) 418

[20] C.R. Münz, J. Resag, B.C. Metsch, H.R. Petry, preprint University of Bonn TK-94-08, nucl-th 9406035, submitted to Phys.Rev. C

[21] J.Resag, C.R.Münz, Preprint University of Bonn TK-94-12, nucl-th 9407034, submitted to Nucl.Phys. A 
[22] J.F. Lagaë: Phys. Rev. D45 (1992) 305, 317

[23] H. Hersbach: Phys. Rev. A46 (1992) 3657, D47 (1993) 3027, Utrecht THU93/13 (1993)

[24] E. Hummel, J. Tjon: Phys.Rev. C42, 423 (1990); G. Rupp, J.A. Tjon, Phys.Rev. C41, 472 (1990); P.C. Tiemeijer, J.A. Tjon: Phys.Lett. B277, 38 (1992); Phys. Rev. C49 (1993) 494, C48 (1994) 896

[25] Yu.L. Kalinovsky, C. Weiss: Z.Phys. C 63 (1994) 275

[26] V.O. Galkin, A.Yu. Mishurov, R.N. Faustov: Sov. J. Nucl. Phys. 55 (1992) 1207, R.N. Faustov, V.O. Galkin, A.Yu. Mishurov: J. Nucl. Phys. 55 (1992) 1080 (englisch: JINR print Dubna, Russia E2-91-451)

[27] M. Neubert: SLAC-PUB-6263, June 1993 (to appear in Phys. Rep.)

[28] N. Isgur, M. B. Wise, Phys. Lett. B 232, (1989) 113; B 237, (1990) 527

[29] A. Wambach: Z. Phys. C 63 (1994) 5697

[30] T. Kugo, M.G. Mitchard, Y. Yoshida: Prog. Theor, Phys. 91 (1994) 5217

[31] T. Murota, Progr.Theor.Phys. 69, 181 (1983), ibid. 1498

[32] Particle Data Group: Phys. Rev. D 50 Part I (1994) 1173

[33] S. Mandelstam, Proc. Roy. Soc. 233, 248 (1955)

[34] D. Lurie, Particles and Fields, Interscience Publishers, 1968

[35] H. Albrecht et al., ARGUS Coll: Z. Phys. C - Particles and Fields 57 (1993) 533

[36] J.G. Körner, G.A. Schuler: Z. Phys.- Particles and Fields C 38, (1988) 511[E: C 41, (1989) 690]; C 46, (1990) 93

[37] D.G. Cassel, CLEO Coll.: (private communication)

[38] S. Sanghera et al., CLEO Coll.: Phys. Rev. D 47, (1993) 791 - 798

[39] G. Altarelli, L. Maiani: Phys. Lett. B52 (1974) 351; M.K. Gaillard, B.W. Lee: Phys. Rev. Lett. 33 (1974) 108

[40] J.L. Rosner: Phys. Rev. D42 (1990) 3732

[41] H. Albrecht et al., ARGUS Coll.: Z. Phys. C - Particles and Fields 54 (1992) 1 
[42] M.E. Luke: Phys. Lett. B 252 (1990) 447

[43] M.A. Shifman, A.I. Weinstein, V.I. Zakharov: Nucl. Phys. B147 (1979) 382, 448

[44] M. Neubert, V. Rieckert, B.Stech, Q.P. Xu: Heavy Flavours, A.J. Buras and M. Lindner eds. (Singapore 1992) p. 286.

[45] G. 't Hooft: Phys. Rev. D 14 (1976) 3432

[46] M. Bauer, B.Stech, and M. Wirbel, Z. Phys. C - Particles and Fields 34 (1987) 103; M. Wirbel, Prog. in Nucl. Part. Phys. 22 (1988) 33

[47] J.D. Bjorken, Nucl. Phys. B (Proc. Suppl.) 11 (1989) 325

[48] A.N. Kamal, Q.P. Xu, and A. Czarnecki, preprint Alberta Thy-3-93 